\title{
Videogrammetric Model Deformation Measurement Technique
}

\author{
A. W. Burner ${ }^{\dagger}$ and Tianshu Liu ${ }^{\ddagger}$ \\ NASA Langley Research Center \\ Hampton, Virginia 23681-2199 \\ $\dagger$ Research Scientist, Senior Member of AIAA, NASA Langley Research \\ Center, MS 236, Hampton, VA 23681-2199 \\ $¥$ Research Scientist, Member of AIAA, NASA Langley Research Center, \\ MS 238, Hampton, VA 23681-2199
}

\begin{abstract}
The theory, methods, and applications of the videogrammetric model deformation (VMD) measurement technique used at NASA for wind tunnel testing are presented. The VMD technique, based on non-topographic photogrammetry, can determine static and dynamic aeroelastic deformation and attitude of a wind-tunnel model. Hardware of the system includes a video-rate CCD camera, a computer with an image acquisition frame grabber board, illumination lights, and retroreflective or painted targets on a wind tunnel model. Custom software includes routines for image acquisition, target-tracking/identification, target centroid calculation, camera calibration, and deformation calculations. Applications of the VMD technique at five large NASA wind tunnels are discussed.
\end{abstract}

\section{Introduction}

The videogrammetric model deformation (VMD) measurement technique is an optical method for measuring aeroelastic deformation and attitude of a model during aerodynamic testing. Based on the principles of close-range photogrammetry, the VMD technique is used to determine the spatial coordinates of targets on a model surface from the target centroids in a series of images. From these spatial coordinates, the model deformation induced by aerodynamic loading is computed. Model deformation may be defined as the change in shape of a wind tunnel model (particularly the wings and control surfaces) under aerodynamic loading. This change in the design geometry can cause differences between the acquired and computational predictions of wind tunnel results if the predictions are based upon rigid body assumptions. Therefore, it is advantageous to measure deformations of wind tunnel models in order to properly compare computational fluid dynamics (CFD) predictions to experimental measurements, particularly in high Reynolds number facilities where dynamic pressure is typically higher than for other facilities. In addition, it is essential to calibrate and validate computational methods such as the finite element method (FEM) with experimental measurements of deformation in order to ensure accurate predictions.

The model deformation measurement capability at NASA includes both single-camera and multiple-camera videogrammetric measurement systems, with emphasis on the measurement of the change of wing twist due to aerodynamic loading [1-3]. A description of the automation of the videogrammetric model deformation technique, experimental procedure and data reduction, description of software, and targeting considerations are given in reference 4. Examples of variations of the model 
deformation technique used for the measurement of angle of attack, sting bending, and the effect of varying model injection rates are presented in reference 5.

A comparison of the VMD measurement technique with other methods for deformation measurements such as projection moiré interferometry [6] and the LED-tracking photogrammetric system made by Northern Digital known as Optotrak [7,8] can be found in reference 9. An electrooptical deflection measurement system developed by Grumman [10] has been used at NASA Dryden for flight tests [11]. The Optotrak system and the Grumman system use synchronized LEDs as active targets. Rotating blade deformation measurements have been made at NASA Glenn [12] with a nonintrusive optical system consisting of a photodiode and a single laser that is used to illuminate the leading and trailing edges of the blade. Model deformation measurements have been made with stereo observations with camera measurement systems at ONERA in France [13, 14]. Optical fibers and quadrant light detectors in addition to a polarization torsionometer have also been used in the past at ONERA for model attitude and deformation measurements [15].

The technical aspects of the VMD measurement technique are described, including nontopographic photogrammetry, image acquisition, target-tracking/identification, target centroid calculation, camera calibration, and deformation calculation. Hardware components and requirements for large wind tunnels are also discussed. Typical applications of the VMD measurement technique in NASA wind tunnels are presented.

\section{The Collinearity Relation between Image Plane and Object Space}

In the VMD image-based measurement technique, data is extracted from two-dimensional (2D) images and then mapped into three-dimensional (3D) object space. Photogrammetry provides a relationship (known as collinearity) between 3D coordinates in object space and corresponding 2D coordinates in images $[16,17]$. The collinearity equations relating the target location $(X, Y, Z)$ in object space to the corresponding point $(x, y)$ in the image plane are

$$
\begin{aligned}
& x-x_{p}-d x=-c \frac{m_{11}\left(X-X_{c}\right)+m_{12}\left(Y-Y_{c}\right)+m_{13}\left(Z-Z_{c}\right)}{m_{31}\left(X-X_{c}\right)+m_{32}\left(Y-Y_{c}\right)+m_{33}\left(Z-Z_{c}\right)}, \\
& y-y_{p}-d y=-c \frac{m_{21}\left(X-X_{c}\right)+m_{22}\left(Y-Y_{c}\right)+m_{23}\left(Z-Z_{c}\right)}{m_{31}\left(X-X_{c}\right)+m_{32}\left(Y-Y_{c}\right)+m_{33}\left(Z-Z_{c}\right)} .
\end{aligned}
$$

In Eq. (1), a parameter set $\left(c, x_{p}, y_{p}\right)$ is the interior orientation of a camera, where $c$ is the principal distance of the lens, $x_{p}$ and $y_{p}$ are the principal-point coordinates on the image plane. Another parameter set $\left(\omega, \phi\right.$, $\left.\hat{\mathrm{e}}, X_{c}, Y_{c}, Z_{c}\right)$ is the exterior orientation of a camera, where $(\omega, \phi, \kappa)$ are the Euler rotational angles and $\left(X_{c}, Y_{c}, Z_{c}\right)$ are the coordinates of the perspective center in object space. The coefficients $m_{i j}(i, j=1,2,3)$ are the rotation matrix elements that are functions of $(\omega, \phi, \kappa)$,

$$
\begin{aligned}
& m_{11}=\cos \phi \cos \kappa, \quad m_{12}=\sin \omega \sin \phi \cos \kappa+\cos \omega \sin \kappa, \\
& m_{13}=-\cos \omega \sin \phi \cos \kappa+\sin \omega \sin \kappa, \quad m_{21}=-\cos \phi \sin \kappa, \\
& m_{22}=-\sin \omega \sin \phi \sin \kappa+\cos \omega \cos \kappa, \quad m_{23}=\cos \omega \sin \phi \sin \kappa+\sin \omega \cos \kappa, \\
& m_{31}=\sin \phi, \quad m_{32}=-\sin \omega \cos \phi, \quad m_{33}=\cos \omega \cos \phi .
\end{aligned}
$$

The terms $d x$ and $d y$ in Eq. (1) are the image coordinate shifts induced by lens distortion. The lens distortion terms can be modeled by the sum of the radial distortion and decentering distortion [18], 


$$
d x=d x_{r}+d x_{d} \text { and } d y=d y_{r}+d y_{d}
$$

where

$$
\begin{aligned}
& d x_{r}=K_{1}\left(x^{\prime}-x_{p}\right) r^{2}+K_{2}\left(x^{\prime}-x_{p}\right) r^{4}, \\
& d y_{r}=K_{1}\left(y^{\prime}-y_{p}\right) r^{2}+K_{2}\left(y^{\prime}-y_{p}\right) r^{4}, \\
& d x_{d}=P_{1}\left[r^{2}+2\left(x^{\prime}-x_{p}\right)^{2}\right]+2 P_{2}\left(x^{\prime}-x_{p}\right)\left(y^{\prime}-y_{p}\right), \\
& d y_{d}=P_{2}\left[r^{2}+2\left(y^{\prime}-y_{p}\right)^{2}\right]+2 P_{l}\left(x^{\prime}-x_{p}\right)\left(y^{\prime}-y_{p}\right), \\
& r^{2}=\left(x^{\prime}-x_{p}\right)^{2}+\left(y^{\prime}-y_{p}\right)^{2} .
\end{aligned}
$$

Here, $K_{1}$ and $K_{2}$ are the radial distortion parameters, $P_{1}$ and $P_{2}$ are the decentering distortion parameters, and $x^{\prime}$ and $y^{\prime}$ are the undistorted coordinates on the image plane. If the image plane is not perpendicular to the optical axis of the lens, the principal-point in (3) is replaced with the point of symmetry for distortion. The $3^{\text {rd }}$ order radial distortion can be either barrel $\left(K_{1}<0\right)$ or pin-cushion $\left(K_{2}\right.$ $>0$ ) distortion. For barrel distortion the image is displaced toward the principal-point, whereas for pincushion distortion the image is displaced away from the principal-point. When the lens distortion is small, the unknown undistorted coordinates can be approximated by the known distorted coordinates, i.e., $x^{\prime} \approx x$ and $y^{\prime} \approx y$. For large distortion, an iterative procedure has to be employed to determine the appropriate undistorted coordinates to improve the estimate. The following iterative relations are used $\left(x^{\prime}\right)^{0}=x$ and $\left(y^{\prime}\right)^{0}=y, \quad\left(x^{\prime}\right)^{k+1}=x+d x\left[\left(x^{\prime}\right)^{k},\left(y^{\prime}\right)^{k}\right]$ and $\left(y^{\prime}\right)^{k+1}=y+d y\left[\left(x^{\prime}\right)^{k},\left(y^{\prime}\right)^{k}\right]$, where the iteration index is $k=0,1,2 \cdots$.

The camera parameters $\left(\omega, \phi, \kappa, X_{c}, Y_{c}, Z_{c}\right)$ and $\left(c, x_{p}, y_{p}, K_{1}, K_{2}, P_{1}, P_{2}, S_{h} / S_{v}\right)$ can be determined from camera calibration, where the additional parameter $S_{h} / S_{v}$ is the ratio between the horizontal and vertical pixel spacings for a digital image. When the camera parameters are known, the image-plane coordinates $(x, y)$ can be transformed to object-space coordinates $(X, Y, Z)$ using the collinearity equations. In order to obtain deformation in three dimensions $(X, Y, Z)$, multiple cameras are needed since there are only two independent equations for three unknowns. For the single-camera implementation of the VMD technique, one of the coordinates $(X, Y, Z)$ of the targets must be given to calculate the remaining two coordinates. The known coordinate for the single-camera VMD technique is typically the semispan coordinate of a row of targets placed in the streamwise direction.

\section{VMD Hardware}

Figure 1 shows a schematic of a single-camera VMD measurement system that includes a CCD camera, a computer with a image acquisition frame grabber board, light source and targets distributed on a model. The sub-components of the VMD system are described below.

\subsection{Cameras}

A broad spectrum of CCD cameras is available, from scientific-grade CCD cameras to standard video-rate CCD cameras. At the low-cost end of the spectrum, standard CCD cameras with interlaced analog outputs have been used routinely for model deformation measurements in wind tunnels. However, one disadvantage of these cameras is blurred imaging of a moving object such as a vibrating wing, due to the fact that the two fields making up an interlaced video frame are exposed at different times. This disadvantage is eliminated (but the spatial resolution in the vertical dimension is reduced 
by $1 / 2$ ) by analyzing video fields instead of video frames. Recently, progressive-scan CCD cameras have been used for VMD measurements in order to maintain vertical resolution, without the added complication of interlaced video fields. It is expected that scientific-grade CCD camera with a gray scale of from 10 to 12 bits and with a framing rate of at least $30 \mathrm{~Hz}$ will be utilized in future enhancements.

\subsection{Image acquisition frame grabber board and computer}

A video image acquisition frame grabber board, either with on-board memory or utilizing system memory, is used to digitize a series of images from a video-rate CCD camera. For targettracking a live video stream is digitized into main system memory and tracked in real-time at $15-\mathrm{Hz}$. Upon receipt of a trigger centroids are determined and are output to a file for every other frame. An alternative to target-tracking is the use of blob analysis to automatically find targets in the field of view after a series of images are digitized. After blob analysis of the image series, centroid calculations with background removal, photogrammetry to determine $X, Z$ coordinates, and computations of deformation and attitude can be accomplished before a trigger for the next data point is received, to enable a truly automated measurement system. However, the target-tracking version of the VMD technique is more robust when spurious glints appear in the field-of-view and where lighting is difficult to control, but requires user intervention if target track is lost, such as occurs when a model is rolled $180^{\circ}$.

\subsection{Targets and Lighting}

Targets are placed on a model surface at locations where deformation measurements are desired. The distribution of the targets is dependent upon whether the single-camera or multiple-camera photogrammetric methods are used. For a single-camera VMD measurement system the targets should be placed in rows on the wing (Fig. 2) at known spanwise location in order to obtain a solution of the collinearity equations with 2 equations in 2 unknowns $(X, Z)$. Both retro-reflective targets and white diffuse polished paint targets have been used. The retro-reflective targets yield a high contrast image when a light source is placed near the camera and are the targets of choice if aerodynamic considerations will allow the additional thickness and roughness. The white diffuse polished paint targets require a dark background to achieve high contrast. A black wall seen in reflection from highly polished metal models produces sufficient contrast of the white diffuse targets, although the contrast and ability to discriminate false targets is much less than for retro-reflective targets. Ideally the thickness of the targets should be small to reduce their intrusiveness to flow. Retroreflective targets are typically 0.004 inch thick, with a surface roughness of $200 \mu$ inch, whereas the polished paint diffuse targets are typically 0.0005 inch thick with a surface roughness of less than $10 \mu \mathrm{inch}$. Ordinary lights (non-laser) can provide sufficient illumination for retro-reflective (when placed near the camera) and for white diffuse targets. In addition to retro-reflective and white targets, black targets have also been used, particularly when the VMD technique was used simultaneously with pressure-sensitive and temperature-sensitive paints. Model deformation data can be used not only to understand the aeroelastic properties of the model, but can also be used to generate a deformed surface grid of the model for improved CFD calculation and PSP mapping.

\section{Software}

Software for the VMD technique includes a suite of routines for image acquisition, targettracking, identification and blob analysis, centroid calculation, camera calibration, photogrammetry, and deformation calculation. The structure of the custom software is shown in Fig. 3. The software is 
used to acquire images, locate targets and calculate their centroids, convert target centroids to spatial coordinates in object space, and compute deformation. Camera calibration provides the interior and exterior orientation parameters of a camera necessary for solution of the perspective collinearity relationship between object space and image space.

\subsection{Image Acquisition}

For the VMD technique used in major NASA wind tunnels, standard analog video cameras and PC-based image acquisition frame grabber boards are utilized. The standard RS-170 video signal has an interlaced analog format, with a vertical resolution of 240 pixels per field, and a horizontal resolution determined by the frame grabber. Horizontal resolutions of 640 or 752 are commonly used. However, two fields are combined to give a total vertical resolution of 480, with the added complication that adjacent rows in the final image may have been exposed at different times. This potential problem (for dynamic situations) can be avoided by using single video fields in the modeldeformation measurement process, but with reduced vertical resolution. Progressive-scan cameras are non-interlaced and may be more suitable for dynamic conditions.

The target-tracking implementation of the VMD technique uses a double-buffer strategy for image acquisition and processing. At the completion of a single image acquisition, a new image is strored in a second buffer at the same time the first buffer is processed. Since the frame grabber board employs a bus-mastering PCI interface, the main processor of the host computer is free to perform the processing even while a grab is in progress. On the completion of each frame, the acquisition and processing operations are switched between the two buffers. In the free-running mode, the bufferswitching and processing operations are triggered automatically by the end of a video frame through the use of callback functions. The double-buffer method implemented in software yields a throughput of 15 frames per second using standard video-rate CCD cameras. With a high-speed progressive-scan CCD camera, a system throughput of 60 images per second is possible.

\subsection{Target-Tracking/Identification and Centroid Calculation}

Once a video frame has been acquired, the targets must be identified and located. This is done with a gray-scale centroid calculation to sub-pixel resolution after subtracting an automatically determined background level in the neighborhood of each target. The target centroid $(\bar{x}, \bar{y})$ is defined

as $\bar{x}=\sum \sum x_{i} I\left(x_{i}, y_{i}\right) / \sum \sum I\left(x_{i}, y_{i}\right)$ and $\bar{y}=\sum \sum y_{i} I\left(x_{i}, y_{i}\right) / \sum \sum I\left(x_{i}, y_{i}\right)$, where $I\left(x_{i}, y_{i}\right)$ is the gray level. While target tracking, only regions in the image plane in the immediate neighborhood of the targets are utilized, which reduces problems associated with stray image features away from the tracking regions.

Once target-tracking is initiated by the user, the system will continuously track the position of the selected targets, returning a live stream of target position data upon trigger. The size parameters and thresholds are maintained in real time for each target, so the system is relatively insensitive to lighting changes that may occur with changes in model attitude. Anomalies in target-tracking may result from bright surface reflections which interfere with a particular target in the image, high-speed model motion which blurs the targets, or loss of image due to severe lighting changes or obstruction in the viewing field. Target-tracking is improved for such cases by memorizing the last "good" position of targets in the target-tracking process. Once tracking is lost on a particular target, the system will recover the target based on memory of the last known position of the lost target. The memory-based target tracking technique has been implemented and significantly enhances the robustness of the targettracking against the anomalies. 


\subsection{Camera Calibration}

Camera calibration is a key element of videogrammetric measurements. Analytical camera calibration techniques have been used to solve the collinearity equations (1) for determination of interior and exterior orientation parameters and lens distortion parameters of the camera/lens system $[19,20]$. Since Eq. (1) is non-linear for the orientation and additional parameters, the iterative method of least-squares estimation has been used as a standard technique for solution of the collinearity equations in photogrammetry. However, direct recovery of the interior orientation parameters including $\left(c, x_{p}, y_{p}\right)$ is often impeded by inversion of a singular or ill-conditioned normal equation matrix which mainly results from strong correlation between the exterior and interior orientation parameters. In order to reduce the correlation between these parameters and enhance the determinability of $\left(c, x_{p}, y_{p}\right)$, the use of multiple camera stations, varying image scales, different camera roll angles and a well-distributed target field in three dimensions has been suggested by Fraser $[21,22]$. These schemes for selecting suitable calibration geometry improve the properties of the normal equation matrix. For applications in wind tunnels where optical access and preparation time are limited, however, an automatic, single-image method of on-the-job camera calibration is desirable that is simple to implement and less time consuming in order to have a minimum impact on productivity [23].

One of the more popular methods in the machine vision community is known as the Direct Linear Transformation (DLT). The DLT equations, originally proposed by Abdel-Aziz and Karara [24], can be obtained by rearranging the collinearity equations and combining terms into a new set of variables,

$$
x+d x=\frac{L_{1} X+L_{2} Y+L_{3} Z+L_{4}}{L_{9} X+L_{10} Y+L_{11} Z+1} \text { and } y+d y=\frac{L_{5} X+L_{6} Y+L_{7} Z+L_{8}}{L_{9} X+L_{10} Y+L_{11} Z+1},
$$

where $L_{k}(k=1$ to 11$)$ are the DLT parameters which are related to the camera orientation parameters. When the lens distortion terms $d x$ and $d y$ are neglected, the DLT equations (4) are linear for the DLT parameters and can be solved directly by a linear least-squares method without an initial guess. Because of its simplicity, the DLT is widely used in both close-range photogrammetry and machine vision. However, when the lens distortion cannot be ignored, the iterative solution method must be used such that the DLT loses the simplicity. Also, the standard DLT gives poor estimates of the principal-point location even when the lens distortion is small. The value of the DLT is that it offers initial approximations of the orientation parameters for more accurate methods.

An optimization method developed by Liu et al. [23] contains two interacting procedures, leastsquares estimation for the exterior orientation parameters and optimization for the interior orientation and other parameters. The optimization method can give, with reasonable precision, the exterior orientation parameters $\left(\omega, \phi, \kappa, X_{c}, Y_{c}, Z_{c}\right)$, and the interior orientation and lens distortion parameters $\left(c, x_{p}, y_{p}, K_{1}, K_{2}, P_{1}, P_{2}, S_{h} / S_{v}\right)$ from a single image of a step calibration target plate (Fig. 4). Here an additional parameter $S_{h} / S_{v}$ is the ratio between the horizontal and vertical pixels for a digitized image. The optimization method (combined with DLT for exterior orientation start values) allows automatic camera calibration for the interior and exterior orientation parameters and additional parameters. This feature particularly facilitates VMD measurements in large production wind tunnels due to time constraints during videogrammetric setup and calibration. The optimization method has 
been used to calibrate a number of CCD cameras with different lenses. Calibration results are in good agreement with measurements by optical techniques.

Besides the analytical camera calibration methods, optical laboratory techniques are available for camera calibration $[25,26]$. These include the laser illumination technique to determine the photogrammetric principal point and point of symmetry for distortion, the displaced reticle technique to determine horizontal and vertical pixel spacing, and space resection combined with linear least squares solutions to determine radial and decentering distortion terms.

\subsection{Deformation calculation}

The data-reduction procedures for the VMD technique include object-space coordinate calculations and deformation calculations. Once the target centroids are computed and the camera orientation parameters are determined, the image-plane coordinates $(x, y)$ can be converted to objectspace coordinates $(X, Y, Z)$ using the collinearity equations. A solution for $(X, Y, Z)$ is not possible using a single set of image coordinates $(x, y)$ unless additional information is available. In a single-camera VMD system, the spanwise locations of the targets are usually fixed to reduce the number of unknowns and to calculate the remaining two coordinates. In other words, the collinearity equations can be solved for $(X, Y, Z)$ under a constraint $Y=$ const. (normally the semispan coordinate). The geometric explanation of the single-camera solution is illustrated in Fig. 5. The solution $(X, Y, Z)$ is the intersection point between the plane $Y=$ const. and a line from the image point passing through the perspective center of the lens. When the angle between the plane $Y=$ const. and optical axis is zero, there is no unique solution. Therefore, this angle must be large enough $\left(>20^{\circ}\right)$ to obtain an accurate single-camera solution. The single-camera approach works very well for pitch-only sweeps, where a streamwise row of the targets on a wing will basically remain at the same spanwise location, but would fail or require additional information, for yaw sweeps. This method is also directly applicable to the angle-of-attack measurements and bending measurements on high-lift systems. Although a two-camera system enables a more direct solution in more general cases, the single-camera approach has an advantage in simplicity. For a two-camera system, simultaneous images are acquired using two video cameras viewing the same set of targets. Thus, for each target, two sets of collinearity equations are sufficient to determine the spatial coordinates $(X, Y, Z)$ based on two sets of image coordinates $(x, y)$ from the two cameras. The least-squares method is used to solve the four equations for three unknown coordinates $(X, Y, Z)$.

Two methods are used to calculate twist and bending of a wing. One is the linear fitting method used only for twist calculation. The local angle-of-attack (AOA), defined as $\grave{e}=-\tan ^{-1}(\ddot{\mathrm{A} Z} / \ddot{\mathrm{A}} X)$, is calculated by a linear fit to the target coordinates in the $(X, Z)$ plane at a given spanwise location, as shown in Fig. 6. In the wind-tunnel coordinate system $(X, Y, Z)$, the $X$-axis is in the flow direction, the $Z$-axis is in the upward direction on the wing surface, and the $Y$-axis is in the spanwise direction following the right-hand rule. The local wing twist due to aerodynamic load is defined as

$$
\text { twist }=\grave{e}_{\text {twist }}=\grave{e}_{\text {on }}(\eta)-\grave{e}_{\text {off }}(\eta) \text {, }
$$

where $\grave{e}_{o n}(\eta)$ and $\grave{e}_{\text {off }}(\eta)$ are the local AOAs in the wind-on and wind-off cases at the normalized semi-span location $\eta$. In practical wind tunnel tests, direct calculation using (5) is not applicable since the model may not be at the same pitch angle in the wind-on and wind-off cases. Thus, a correction

method is used for twist calculation. First, the angle difference $\ddot{A} \grave{e}\left(\grave{e}_{\text {off }}\right)=\grave{e}_{\text {off }}-\grave{a}_{r e f}$ in the wind-off 
case is fitted using a polynomial as a function of $\grave{e}_{\text {off }}$, where á ${ }_{r e f}$ is the reference angle-of-attack and $\grave{e}_{\text {off }}$ is the local AOA obtained by the VMD system in the wind-off case. The reference á ${ }_{r e f}$ could be the AOA readout from a wind tunnel system or other reliable AOA data. The corrected AOA ( $\left.\grave{e}_{\text {on }}\right)_{\text {corr }}$ in the wind-on case is calculated using $\left(\grave{e}_{\text {on }}\right)_{c o r r}=\grave{e}_{o n}-\ddot{A} \grave{e}\left(\grave{e}_{o n}\right)$. Finally, the wing twist is obtained using the following relation

$$
\text { twist }=\grave{e}_{\text {twist }}=\left(\grave{e}_{\text {on }}\right)_{\text {corr }}-\grave{a}_{\text {ref }} \text {. }
$$

A transformation method can be used to calculate both wing twist and bending. Assuming that the cross-section of a wing does not deform, a conformal transformation between the wind-on and wind-off coordinates $\left(X_{o n}, Z_{o n}\right)$ and $\left(X_{o f f}, Z_{\text {off }}\right)$ is

$$
\left(\begin{array}{l}
X_{o n} \\
Z_{\text {on }}
\end{array}\right)=\left(\begin{array}{cc}
\cos \grave{e}_{\text {twist }} & \sin \grave{e}_{\text {twist }} \\
-\sin \grave{e}_{\text {twist }} & \cos \grave{e}_{\text {twist }}
\end{array}\right)\left(\begin{array}{l}
X_{\text {off }} \\
Z_{\text {off }}
\end{array}\right)+\left(\begin{array}{c}
T_{x} \\
T_{z}
\end{array}\right),
$$

where $T_{x}$ and $T_{z}$ are the translations in the $X$ - and $Z$-directions, respectively. Given the coordinates $\left(X_{o n}, Z_{o n}\right)$ and $\left(X_{o f f}, Z_{o f f}\right)$ of a number of targets, the twist $\grave{e}_{\text {twist }}$ and translations $T_{x}$ and $T_{z}$ can be determined using a least-squares method. Wing bending is

$$
\text { bending }=T_{z}-\left(T_{z}\right)_{\text {ref }} \text {, }
$$

where $\left(T_{z}\right)_{\text {ref }}$ is the reference $Z$-translation in a reference location such as the fuselage.

\section{Uncertainty}

The uncertainty of the VMD technique is related to the uncertainties in target centroid measurements, camera calibration, and data-reduction (calculations of coordinates, twist and bending). The uncertainty in target centroid measurement is associated with camera noise, centroid calculation schemes, target size, and spatial quantization of a CCD sensor. The random errors associated with the camera noise can be collectively represented by the centroid variations for spatially fixed targets. Statistics of the target centroid variations have been measured using a standard video-rate CCD camera with a $75 \mathrm{~mm}$ lens viewing an array of 1/4-in. diameter circular targets. Figure 7 shows typical histograms of the centroid variations in the horizontal $(x)$ and vertical $(y)$ coordinates on the image plane. The standard deviations of the centroid variations in the $x$ - and $y$-directions in images are 0.0081 and 0.0043 pixels, respectively, for the CCD camera with a format of $640 \times 480$ pixels. The centroid uncertainties limit the accuracy of VMD measurements in the object space. When the image plane is approximately parallel to the $(X, Y)$ plane in the object space, estimates of the limiting uncertainties in the spatial coordinates associated with the centroid random variations are $\left(\delta X / L_{X}\right)_{\min }=0.0081 / 640=1.3 \times 10^{-5}$ and $\left(\delta Y / L_{Y}\right)_{\min }=0.0043 / 480=0.9 \times 10^{-5}$. For example, when the characteristic lengths in the object space are $L_{X}=7 \mathrm{in}$. and $L_{Y}=9 \mathrm{in}$., the corresponding measured length differences are $(\delta X)_{\min }=182 \mu \mathrm{in}$ and $(\delta Y)_{\min }=162 \mu \mathrm{in}$.

A bias error occurs in the centroid calculation due to perspective imaging and lens distortion since the center of the target image does not coincide with the geometrical center of the target [27]. This deviation may be as large as $0.3 \%$ of the target diameter and is dependent on the viewing angle of the camera, target size, sensor size, and focal length. Another error in centroid calculation is associated the sensor quantization, which is inversely proportional to the square root of the number of pixels in the target image [27]. 
The uncertainty in camera calibration is associated with the correctness of the mathematical model (1) and the accuracy of the numerical methods to solve it. To examine the accuracy of camera calibration, one can compare the calculated camera orientation and lens distortion parameters with more accurate values obtained using other independent techniques (that are too time consuming or difficult to implement in large production wind tunnels). The aforementioned optimization method for camera calibration has been compared against the optical techniques described by Burner et al. [25, 26]. The optical techniques may be more accurate, but require special laboratory equipment that may not be available or may be unsuitable for on-site calibration. The results obtained by the optimization method are in reasonable agreement with the optical techniques for several lenses [23]. A common figure-ofmerit for camera calibration is represented by the standard deviation of the residuals of the image coordinate calculation. These residuals are a convenient measure for goodness of the least-squares fitting. Typically, the residuals of camera calibration are less than one micron (less than 0.1 pixel) on the CCD array, depending on the accuracy of the given coordinates of a calibration target plate.

The uncertainty in data-reduction includes contributions from calculations of the target coordinates, wing twist, and bending. In a single-camera VMD system, the fixed spanwise locations of the targets $(Y=$ const. $)$ are assumed to be known in order to calculate the remaining two coordinates from the collinearity equations. However, $Y$ is not constant during wind-on conditions due to model yaw dynamics and wing bending. For example, lateral model motion as large as $\pm 3 \mathrm{~mm}$ has been noted at one facility. Recording an image sequence in order to determine mean image coordinates reduces this error due to varying $Y$. In addition, wing bending causes the $Y$ coordinate of wing targets to decrease, which causes a bias error in the computation of $X$ and $Z$. Assuming an approximate 2nd order wing bending, a wing tip deflection of $20 \mathrm{~mm}$, and semispan equal to $580 \mathrm{~mm}$, the change in $Y$ value due to wing bending would be approximately $0.5 \mathrm{~mm}$ for targets at the tip. The shift in $Y$ for targets inboard of the tip would decrease rapidly. Note that targets at the same semispan station will experience only slight differences in both bending and shifts in $Y$ value. In fact, it is this small difference in bending between fore and aft targets which produces wing twist for swept wings under load. For instance, fore and aft targets in the streamwise direction at the wing tip of the previous example would experience a wing twist of almost $-2^{\circ}$ for a $30^{\circ}$ swept-back wing. For two targets at the tip separated by $50 \mathrm{~mm}$ the difference in bending would be $1.7 \mathrm{~mm}$ out of a total bending of $20 \mathrm{~mm}$ with a corresponding difference in the shift of the $Y$ value for the two targets of $0.06 \mathrm{~mm}$. A shift in $Y$ value of $0.06 \mathrm{~mm}$ will cause a difference in image scale between the fore and aft targets of only 1.00003 for typical object distances at wind tunnels $(\sim 1.8 \mathrm{~m})$. The error in angle caused by this small difference in scale is negligible compared to other error sources for this typical example.

The repeatability in VMD measurements (which includes other error sources such as effects of glass window, tunnel vibration, and change of the gas refraction index) was determined with repeat tests at the National Transonic Facility (NTF) [1]. The run-to-run repeatabilities of wing twist measurements of a High-Speed Research model during air runs are presented in Table 1 for $M=0.3, Q$ $=153$ psf (4 runs with 30 data points per run) and $M=0.9, Q=965$ psf (4 runs with 23 data points per run), where $M$ is Mach numbers and $Q$ is dynamic pressures. Wing twist, $\theta_{t w i s t}$, was computed at normalized semispan stations $0.635,0.778$, and 0.922 using the linear fitting method (6). The mean and maximum of the computed sample standard deviation of each repeat set of four data points are denoted as $\sigma_{\text {mean }}$ and $\sigma_{\text {max }}$ in the tables. The arcsector AOA sensor (ARCSEC) is affected less by dynamics than the onboard accelerometer so that $\sigma_{\text {mean }}$ for the ARCSEC variable may be taken as an indicator of model pitch angle variability for repeat points. These two tables show that the mean 
standard deviation in $\theta_{\text {twist }}$ for repeat points was less that $0.02^{\circ}$ in air mode. In general, the standard deviation of the wing twist, $\theta_{\text {twist }}$, is less than the standard deviation of the angle, $\theta$, since any real variations in angle-of-attack settings between repeat points present in $\theta$ are subtracted out when $\theta_{\text {twist }}$ is computed. However, note that any error and variability in the reference angle of attack for $\alpha_{\text {ref }}$ will be added to the $\theta_{\text {twist }}$ value. A plot of the repeatability versus $\alpha_{\text {ref }}$ presented in Fig. 8 for $\eta=0.922$ shows worse repeatability at higher $\alpha_{\text {ref }}$, especially at the higher Mach number and dynamic pressure $Q$. Data for the other two semispan stations behaved similarly. Comparisons of repeat runs from two tests separated by over five months are presented in Table 2. The mean and standard deviation, $\sigma$, of the differences are presented as a function of semispan location, Mach number, and dynamic pressure. The number of data points used for these comparisons varied from 18 to 26 .

\section{VMD Applications in Wind Tunnels}

Aeroelastic deformation measurements have been made for a number of tests at five large production wind tunnels at NASA. These facilities are the National Transonic Facility (NTF), the Transonic Dynamics Tunnel (TDT), the Unitary Plan Wind Tunnel (UPWT) at NASA Langley, the 12FT Pressure Tunnel at NASA Ames (12 Ft), and the Langley 16-Foot Transonic Tunnel (16 Ft). The location of the data-recording camera varies with the tunnel due to window location constraints, competition with other instrumentation for viewing ports, and ease of mounting. VMD measurements on sting-mounted horizontal models have been made at the NTF, UPWT, 16-Ft, and TDT. VMD measurements have been made on wall mounted semi-span models at the NTF and TDT. Measurements have been made on floor mounted semispan and bipod supported full models at the 12Ft tunnel.

\subsection{National Transonic Facility}

The National Transonic Facility (NTF) is a fan-driven, closed circuit, continuous-flow pressurized wind tunnel. The $8.2 \times 8.2 \times 25$-ft long test section has a slotted-wall configuration. The wind tunnel can operate in an elevated temperature mode up to $\mathrm{T}=140^{\circ} \mathrm{F}$, normally using air, and in a cryogenic mode, using liquid nitrogen as a coolant, to obtain a test temperature range down to about $250^{\circ} \mathrm{F}$. The design total pressure range for the NTF is from 15 psia to 130 psia. The combination of pressure and cold test gas can provide a maximum Reynolds number of $1.2 \times 10^{8}$ at Mach 1.0, based on a chord length of 9.75 inches. These characteristics afford full-scale Reynolds number testing for a wide range of aircraft. A major instrumentation challenge at the National Transonic Facility is the requirement to make measurements over the wide range of temperature from $140^{\circ} \mathrm{F}$ down to $-250^{\circ} \mathrm{F}$. Aeroelastic deformation measurements have been made at the NTF for both High Speed Research (HSR) [1] and Advanced Subsonic Technology (AST) [2] models. Figure 9 shows typical wing twist of a HSR model at different dynamic pressures for $\eta=0.922$. The Mach number and total pressure were varied to give the desired dynamic pressure.

\subsection{Transonic Dynamics Tunnel}

The Langley Transonic Dynamics Tunnel (TDT) is used for aeroelastic research and flutterclearance and other aeroelastic-verification tests of fixed-wing and rotary-wing flight vehicles and launch vehicles. The TDT is a continuous-flow, variable-pressure wind tunnel with a 16-ft by $16-\mathrm{ft}$ test section. The tunnel uses either air or a heavy gas as the test medium and can operate at Mach numbers 
up to about 1.2 while obtaining Reynolds numbers per foot of approximately $3 \times 10^{6}$ in air and $10 \times 10^{6}$ in heavy gas.

The first automated videogrammetric measurements of wing twist and bending at NASA Langley were made at the TDT in 1994 where the application of high contrast targets on the wing made possible the use of image processing techniques to automatically determine the image coordinates of the targets. Image sequence record lengths at a $60 \mathrm{~Hz}$ rate of up to $8 \mathrm{sec}$ per data point have been taken for dynamic studies. Videogrammetry has been used at the TDT for a number of tests of semispan models, both rigid and flexible, and one sting-mounted full model. Measurements have been made on the DARPA/Wright Labs/Northrop Grumman Smart Wing that had variable twist and adaptive control surfaces to provide continuous wing contour and variable camber [28]. Tests were first conducted on a conventional wing model without smart structures for comparison to the Smart Wing and to validate the model deformation system. The system was used to determine the trailing edge deflection angles of the Smart Wing, which were embedded with shape memory alloy (SMA). The system was also used to measure model wing twist when the SMA torque tubes were activated. The system provided near real time model control surface deflections and twist. The measurement system at the TDT has also been adapted for displacement measurements during a test of a piezoelectric wafer actuator to alter the upper surface geometry of a subscale airfoil to enhance performance $[29,30]$.

\subsection{Unitary Plan Wind Tunnel}

The Langley Unitary Plan Wind Tunnel (UPWT) is a closed circuit, continuous-flow, variabledensity tunnel with two 4-ft by 4-ft by 7 -ft test sections. One test section has a design Mach number range from 1.5 to 2.9, and the other has a Mach number range from 2.3 to 4.6. The tunnel has slidingblock-type nozzles that allow continuous variation in Mach number while the facility is in operation. The maximum Reynolds number per foot varies from $6 \times 10^{6}$ to $11 \times 10^{6}$, depending on Mach number. A VMD measurement system has been used at UPWT for aeroelastic studies to assess Mach number and Reynolds number effects in addition to comparisons of models with flapped and solid wings. For example, data for the aerodynamically induced wing twist and bending of an HSR NCV model near the wing tip $(\eta=0.992)$ for Reynolds number sweeps at Mach $=2.4$ are plotted in Fig. 10. Reynolds number variations were obtained by changing the dynamic pressure, thus the plot in Fig. 10 reflects the dynamic pressure effect on the change in aeroelastic wing twist. The maximum wing twist of $-1.25 \mathrm{deg}$ at Mach 2.4 occurs at a Reynolds number of $4.9 \times 10^{6}$. The nearly linear change in twist and displacement as a function of alpha has been observed for a number of HSR models.

\subsection{Ames 12-Ft Pressure Tunnel}

The restored 12-Foot Pressure Tunnel at NASA Ames is a closed-return, variable-density tunnel with a continuously variable Mach number from 0.05 to 0.60 . Maximum Reynolds number is $12 \times 10^{6}$ per foot. The twelve-foot diameter, 28.5-foot long test section has 4-foot wide flats on the ceiling, floor and sidewalls. The 12-Foot tunnel is the only large scale, pressurized, very low turbulence, subsonic wind tunnel in the United States. It provides unique capabilities in high Reynolds number testing for the development of high-lift systems for commercial transport and military aircraft and high angle of attack testing of maneuvering aircraft. Aeroelastic model deformation measurements have been made for full models supported on the bipod and semispan models floor mounted vertically. For the bipod supported model the deformation system viewed retroreflective targets placed at various semispan locations along the right wing and body. The CCD camera was installed for protection in a pressure vessel with window. An incandescent lamp was placed near the camera in the same viewport in order 
to illuminate the retroreflective targets. Part of the wing had to be painted flat black to eliminate specular reflections where the surface normal bisected the camera and light source locations. Aerodynamically induced wing twist and change in vertical displacement near the wing tip $(\eta=0.99)$ versus alpha for the 4\% Arrow Wing HSR model at the Ames 12-Ft Pressure Tunnel are shown in Fig. 11. The Mach number was 0.225 , the Reynolds number per foot was $8.51 \times 10^{6}$, and the dynamic pressure was $435 \mathrm{psf}$. Data for flaps deflected and undeflected are presented, which clearly indicate effects of the flaps on the twist and vertical displacement. In addition, simultaneous tests using the videogrammetric model deformation measurement technique with pressure sensitive paint (PSP) [31] and transition detection using temperature sensitive paint (TSP) [32] were performed at the 12-Ft Tunnel.

\subsection{6-Foot Transonic Tunnel}

The Langley 16-Foot Transonic Tunnel is a single-return atmospheric wind tunnel with a slotted transonic test section and a Mach number range o 0.2 to 1.25. The octagonal test section measures 15.5 $\mathrm{ft}$ across the flats. Models are mounted in the test section by sting, sting-strut, or semispan support arrangements. The dedicated VMD system for the facility is suitable only for sting mounted models at present. The CCD camera, light source and power supply are currently mounted on a movable flat of the test section which must be compensated for with wind-off polars at the various flat angular settings. A flat mirror is used to direct the light from a 150-watt lamp around the camera and out the same window. The light output is variable from the control room. A vortex cooler requiring a pressurized air supply is used to reduce the temperature near the camera, which may reach $170^{\circ} \mathrm{F}$ without cooling. The model center of rotation is located near to the wing area, which enables a smaller field of view in order to increase resolution. Figure 12 shows aerodynamic load induced wing twist versus normalized semispan location for an HSR TCA model tested at Mach 0.6 and Mach 1.1. Data for the baseline configuration without deflected flaps is shown for the angles-of-attack -2, 1, 4, and 7 degrees. Data taken at the test section wall flat settings for the various Mach numbers indicate that the flat setting has little effect on the measured twist, but causes a zero shift in displacement of up to 0.07 inches that varies with semispan station.

\subsection{Orientation and Deformation Measurements of a Probe}

A two-camera VMD system has been used to measure the orientation angles of a seven-hole probe during the calibration in the Probe Calibration Tunnel at NASA Langley. The orientation angles of the seven-hole probe are controlled by two rotational stages. The orientation angles $\alpha$ and $\beta$ of the probe and the coordinate system are shown in Fig. 13. During the calibration, the probe deforms due to aerodynamic load. Thus, the rotation-stage readings do not accurately represent the orientation angles. Two CCD cameras with 35-mm lenses were used to image the probe through a wind-tunnel window. Four retro-reflective targets were attached to the blackened probe surface along the probe centerline. Three-dimensional coordinates of the targets were obtained and the probe orientation angles were calculated from the coordinates of these targets. Measurements were made at Mach numbers $0.4,0.5$, 0.6 , and 0.7 and total pressures 32 and 60 psi. The probe orientation angles $\alpha$ and $\beta$ vary from -50 to 50 degrees. Figure 14 shows the distributions of angle deviations generated by aerodynamic forces of VMD-measured $\alpha$ and $\beta$ from the rotation-stage readings in the parameter space $(\alpha, \beta)$, for a Mach number of 0.5 and the total pressure of 60 psi.

\subsection{Aeroelastic Divergence Measurements of an Airfoil}


Aeroelastic divergence testing of an airfoil section has been conducted in the Flutter Research and Experiment Device (FRED) Wind Tunnel. FRED is an open circuit table-top wind tunnel with a maximum operating velocity of 85 miles per hour. The plexiglass test section of 6 inches by 6 inches provides excellent optical access for model viewing. A rigid wing was mounted to a mechanism that allowed only a pitching motion of the airfoil with a single degree of freedom, governed by a torsional spring. Measurements made using the VMD technique are depicted in Fig. 15 showing the time history of the angle-of-attack (AOA) as the flow velocity was decreased. The elastic angle-of-attack varied as a function of the flow velocity, emulating the development of wing twist as an aircraft changes flight condition. The time history of the AOA of the airfoil shows initial statically unstable, divergent behavior indicated by a maximum airfoil deflection of $-17^{\circ}$ at a limit stop. A stable dynamic mode of the system produced the oscillatory behavior. As the flow velocity was decreased the airfoil became statically stable, indicated by the AOA returning to $1^{\circ}$, which was the prescribed AOA for the experimental setup.

\section{Conclusions}

A videogrammetric technique has been found to be very useful for the measurement of windtunnel model attitude, deformation, and displacement measurements. The single-camera single-view implementation of the VMD technique, even though restricted to pitch polars, offers operational and setup simplicity that make the technique particularly useful in large production wind tunnels. The multi-camera VMD technique can provide 3D information and is not restricted to pitch polars, but has additional setup, viewport, synchronization, and lighting requirements. Model deformation measurements have been made with the single-camera single-view VMD technique at five large NASA wind tunnels. Model deformation data can be used not only to understand the aeroelastic properties of the model, but can also be used to generate a deformed surface grid of the model for more correct CFD calculation and PSP mapping.

\section{Acknowledgments}

The operations and research staff at the NASA Langley NTF, TDT, UPWT, 16-Ft, and the NASA Ames 12-Ft Tunnel are acknowledged for assistance and support in test technique developments and applications at their facilities. L. Owens, R. Wahls, R. Mineck, and G. Erickson from NASA Langley have been associated with a number of model deformation tests at the NTF and the UPWT. R. Radeztsky and S. Garg, formerly with the High Technology Corporation, are acknowledged for their development of the target-tracking videogrammetric model deformation system. W. K. Goad is acknowledged for his many contributions to the dedicated VMD systems at NASA Langley. $H$. Dismond and K. Cate are acknowledged for continuing support in VMD installations, calibration, software development, and operations. B. S. Sealey is acknowledged for improvements and applications of the polished-painted target technique at the NTF. J. Heeg from NASA Langley is acknowledged for the dynamic data plot from the Flutter Research and Experiment Device (FRED) Wind Tunnel.

\section{References}

[1] Burner, A. W., Wahls, R. A., and Goad, W. K., "Wing Twist Measurements at the National Transonic Facility", NASA TM 110229, Feb. 1996.

[2] Hooker, J. R., Burner, A. W., Valla, R.: "Static Aeroelastic Analysis of Transonic Wind Tunnel Models using Finite Element Methods”. AIAA Paper 97-2243, June 1997. 
[3] Burner, A, W, "Model deformation measurements at NASA Langley Research Center," $A G A R D$ Conference Proceedings CP-601: Advanced Aerodynamic Measurement Technology, presented at the 81st Meeting and Symposium of the Fluid Dynamics Panel, Seattle, WA, pp. 34-1 to 34-9, September, 1997.

[4] Burner, A. W. and Martinson, S. D., "Automated wing twist measurements under aerodynamic load," AIAA Paper 96-2253, June 1996.

[5] Burner, A. W., Radeztsky, R. H. and Liu, T., "Videometric applications in wind tunnels," Videometrics V, Proceedings of The International Society for Optical Engineering (SPIE), Vol. 3174, San Diego, California, pp. 234-247, 1997.

[6] Fleming, G. A., Soto, H. L., South, B. W., Bartram, S. M., "Advances in Projection Moiré Interferometry Development for Large Wind Tunnel Applications”, paper \# 1999-01-5598 presented at the SAE 1999 World Aviation Congress and Exposition, San Francisco, CA, October 19-21, 1999.

[7] Watzlavick, R. L, Crowder, J. P., Wright, F. L., "Comparison of Model Attitude Systems: Active Target Photogrammetry, Precision Accelerometer, and Laser Interferometer”, AIAA Paper 96-2252, June 1996.

[8] Willard, P., Hardin, J. D., Whitehead, J. H., "Determination of In-Flight aeroelastic Deformation of a Transport High-Lift System Using Optical Position Measurement Technology", presented at the $1^{\text {st }}$ AIAA Aircraft Engineering, Technology, and Operations Congress, Los Angeles, CA September 19-21, 1995.

[9] Burner, A. W., Fleming, G. A., and Hoppe, J. C., "Comparison of Three Optical Methods for Measuring Model Deformation” AIAA-2000-0835, January 2000.

[10] DeAngelis, V. M., Fodale, R., “Electro-Optical Flight Deflection Measurement System”, presented at the Society of flight Test Engineers Conference, 1987.

[11] Lokos, W. A., "Predicted and Measured In-Flight wing Deformations of a Forward-Swept-Wing Aircraft", NASA TM 4245, Nov. 1990.

[12] Kurkov, A. P., “Optical Measurement of Propeller Blade Deflections”, NASA TP 2841, 1988.

[13] Lamiscare, B., Sidoruk, B., Castan, E., and Bazin, M., "Dispositif RADAC de Mesure des Deformations de Maquette. Premiers Resultats Obtenus dans la Soufflerie F1”, ONERA T.P No. 1990-57, 1990.

[14] Surget, J., "Model Attitude and Deformation Measurement in wind Tunnels", ONERA T.P. No. 1982-91, 1982.

[15] Charpin, F., Armand, C, Selvaggini, R., "Measurement of Model Deformation in Wind Tunnels", ONERA T.P. No. 1986-126, 1986.

[16] McGlone, J. C., "Analytic data-reduction schemes in non-topographic photogrammetry," Chapter 4, Non-Topographic Photogrammetry, 2nd Edition, (H.M. Karara, editor), American Society for Photogrammetry and Remote Sensing, Falls Church, Virginia, pp. 37-55, 1989.

[17] Wong, K. W., "Basic mathematics of photogrammetry," Chapter 2, Manual of Photogrammetry, 4th Edition, (C.C. Slama, editor), American Society of Photogrammetry, Falls Church, Virginia, pp. 37-101, 1980.

[18] Fryer, J. G., "Camera calibration in non-topographic photogrammetry," Chapter 5, NonTopographic Photogrammetry, 2nd Edition, (H.M. Karara, editor), American Society for Photogrammetry and Remote Sensing, Falls Church, Virginia, pp. 59-69, 1989. 
[19] Rüther, H., "An overview of software in non-topographic photogrammetry," Chapter 10, NonTopographic Photogrammetry, 2nd Edition, (H.M. Karara, editor), American Society for Photogrammetry and Remote Sensing, Falls Church, Virginia, pp. 129-145, 1989.

[20] Tsai, R. Y., "A versatile camera calibration technique for high-accuracy 3D machine vision metrology using off-the-shelf TV cameras and lenses," IEEE Journal of Robotics and Automation, Vol. RA-3, No. 4, August, pp. 323-344, 1987.

[21] Fraser, C. S., "Photogrammetric camera component calibration - A review of analytical techniques," Workshop on Calibration and Orientation of Cameras in Computer Vision (TU-1), XVII Congress, International Society of Photogrammetry \& Remote Sensing, Washington, DC, 1992.

[22] Fraser, C. S., "Optimization of networks in non-topographic photogrammetry," Chapter 8, NonTopographic Photogrammetry, 2nd Edition, (H.M. Karara, editor), American Society for Photogrammetry and Remote Sensing, Falls Church, Virginia, pp. 95-106, 1989.

[23] Liu, T., Cattafesta, L., Radezsky. R. and Burner, A. W., "Photogrammetry applied to wind tunnel testing", AIAA J. (in press), 2000.

[24] Abdel-Aziz, Y. I. and Karara, H. M., "Direct linear transformation from comparator coordinates into object space coordinates in close-range photogrammetry," Proc. ASP/UI Symp. on CloseRange Photogrammetry, Univ. of Illinois at Urbana-Champaign, Urbana, Illinois, pp. 1-18, 1971.

[25] Burner, A. W., Snow, W. L., Shortis, M. R. and Goad, W. K., "Laboratory calibration and characterization of video cameras," SPIE Vol. 1395 Close-Range Photogrammetry Meets Machine Vision, pp.664-671, 1990.

[26] Burner, A. W., "Zoom lens calibration for wind tunnel measurements," Videometrics IV, Proceedings of The International Society for Optical Engineering (SPIE), Vol. 2598, Philadelphia, Pennsylvania, pp. 19-33, 1995.

[27] Lenz, R. and Fritsch, D., Accuracy of Videogrammetry with CCD Sensors, ISPRS Journal of Photogrammetry and Remote Sensing, 45, pp. 90-110, 1990.

[28] Fleming, G. A., and Burner, A. W.: "Deformation Measurements of Smart Aerodynamic Surfaces", SPIE International Symposium on Optical Science, Engineering and Instrumentation, July 18-23, 1999, Denver, CO.

[29] Pinkerton, J. L., McGowan, A. R., Moses, R. W., Scott, R. C., Hegg, J., "Controlled Aeroelastic Respose and Airfoil Shaping Using Adaptive Materials and Integrated Systems", presented at the SPIE 1996 Symposium on Smart Structures and Itegrated Systems, San Diego, CA Feb 26-29, 1996.

[30] Pinkerton, J. L. and Moses, R. W., "A Feasibility Study to Control Airfoil Shape Using THUNDER," NASA TM-4767, Nov. 1997.

[31] Bell, J. H. and Burner, A. W: “Data Fusion in Wind Tunnel Testing; Combined Pressure Paint and Model Deformation Measurements (Invited)”, AIAA Paper 98-2500, June 1998.

[32] Burner, A. W., Liu, T., Garg, S., Bell, J.H. and Morgan, D.G., "Unified model deformation and flow transition measurements", Journal of Aircraft, Vol. 36, No. 5, 898-901, 1999. 


\begin{tabular}{|l|l|l|l|l|l|l|l|l|l|}
\hline \multicolumn{3}{|c|}{ Spanwise Location $(\eta)$} & \multicolumn{2}{c|}{0.635} & \multicolumn{2}{c|}{0.778} & \multicolumn{2}{c|}{0.922} \\
\hline & & $\Delta A R C S E C$ & $\Delta \alpha_{\text {on }}$ & $\Delta \theta_{\text {twist }}$ & $\Delta \theta$ & $\Delta \theta_{\text {twist }}$ & $\Delta \theta$ & $\Delta \theta_{\text {twist }}$ & $\Delta \theta$ \\
\hline $\begin{array}{l}M=0.3 \\
Q=153 \mathrm{psf}\end{array}$ & $\sigma_{\text {mean }}$ & 0.010 & 0.011 & 0.008 & 0.009 & 0.007 & 0.012 & 0.006 & 0.018 \\
\cline { 2 - 10 } & $\sigma_{\text {max }}$ & 0.015 & 0.019 & 0.019 & 0.018 & 0.024 & 0.029 & 0.017 & 0.018 \\
\hline $\begin{array}{l}M=0.9 \\
Q=965 \mathrm{psf}\end{array}$ & $\sigma_{\text {mean }}$ & 0.006 & 0.011 & 0.016 & 0.016 & 0.013 & 0.015 & 0.014 & 0.016 \\
\cline { 2 - 10 } & $\sigma_{\text {max }}$ & 0.012 & 0.015 & 0.029 & 0.032 & 0.025 & 0.033 & 0.037 & 0.051 \\
\hline
\end{tabular}

Table 1. Run-to-run repeatability in degrees for four repeat air runs.

\begin{tabular}{|l|l|c|c|c|c|c|c|}
\hline \multicolumn{2}{|c|}{ Spanwise Location $(\eta)$} & \multicolumn{2}{c|}{0.635} & \multicolumn{2}{c|}{0.778} & \multicolumn{2}{c|}{0.922} \\
\hline \multicolumn{1}{|c|}{$Q$} & $Q(p s f)$ & mean & $\sigma$ & mean & $\sigma$ & mean & $\sigma$ \\
\hline 0.3 & 154 & -0.012 & 0.022 & -0.013 & 0.030 & -0.006 & 0.027 \\
\hline 0.6 & 534 & -0.004 & 0.059 & 0.013 & 0.071 & 0.011 & 0.049 \\
\hline 0.3 & 805 & 0.022 & 0.093 & 0.017 & 0.109 & 0.026 & 0.087 \\
\hline 0.9 & 967 & 0.001 & 0.027 & -0.016 & 0.026 & -0.005 & 0.047 \\
\hline
\end{tabular}

Table 2. Test-to-test repeatability in degrees during air runs. Units for dynamic pressure $Q$ are psf. 


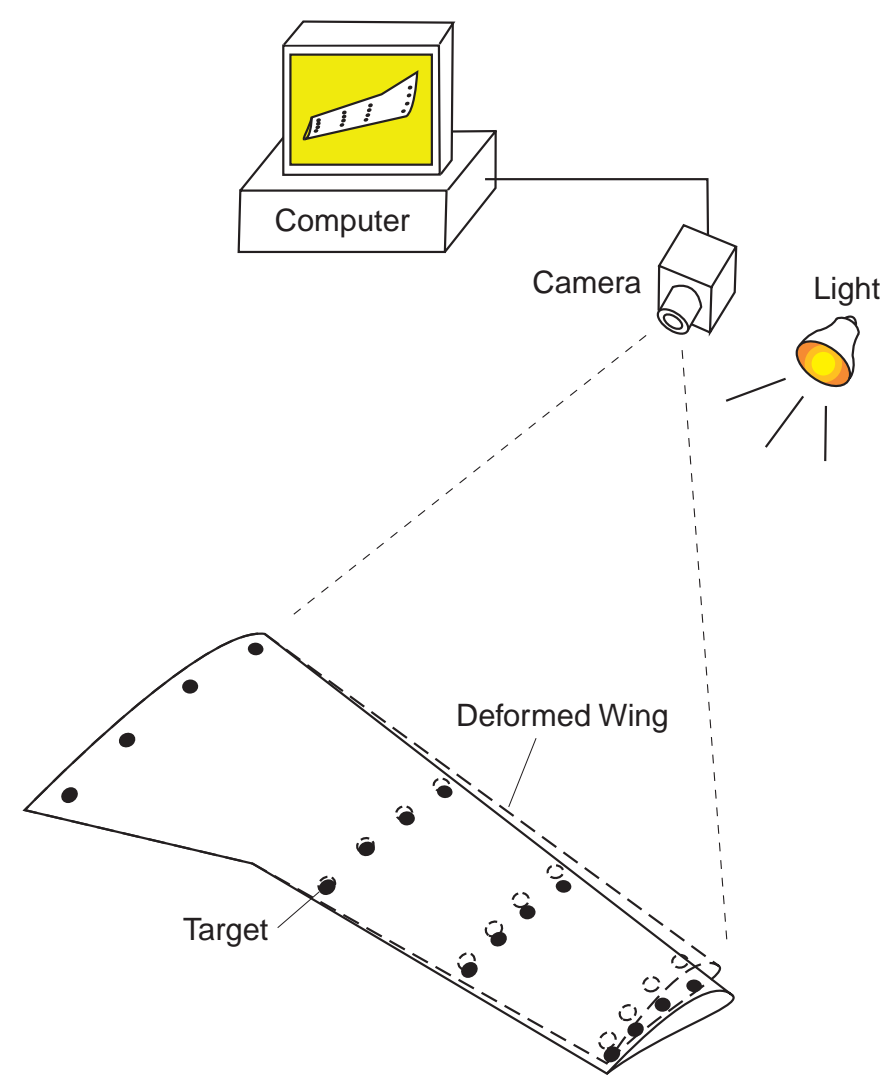

Figure 1. Schematic of a single-camera VMD system 


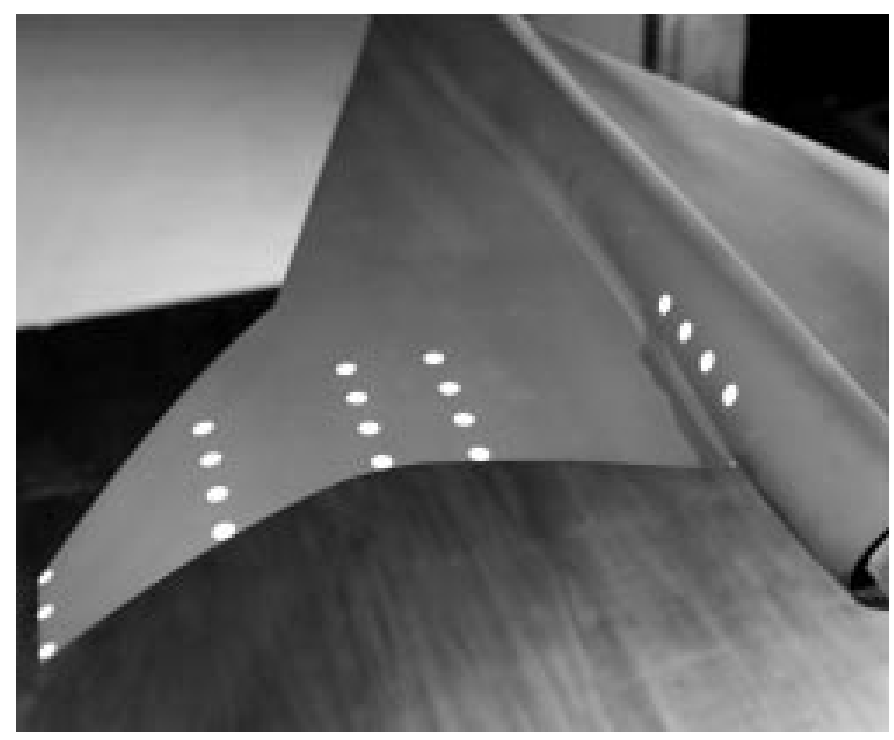

Figure 2. Target rows on a model. 


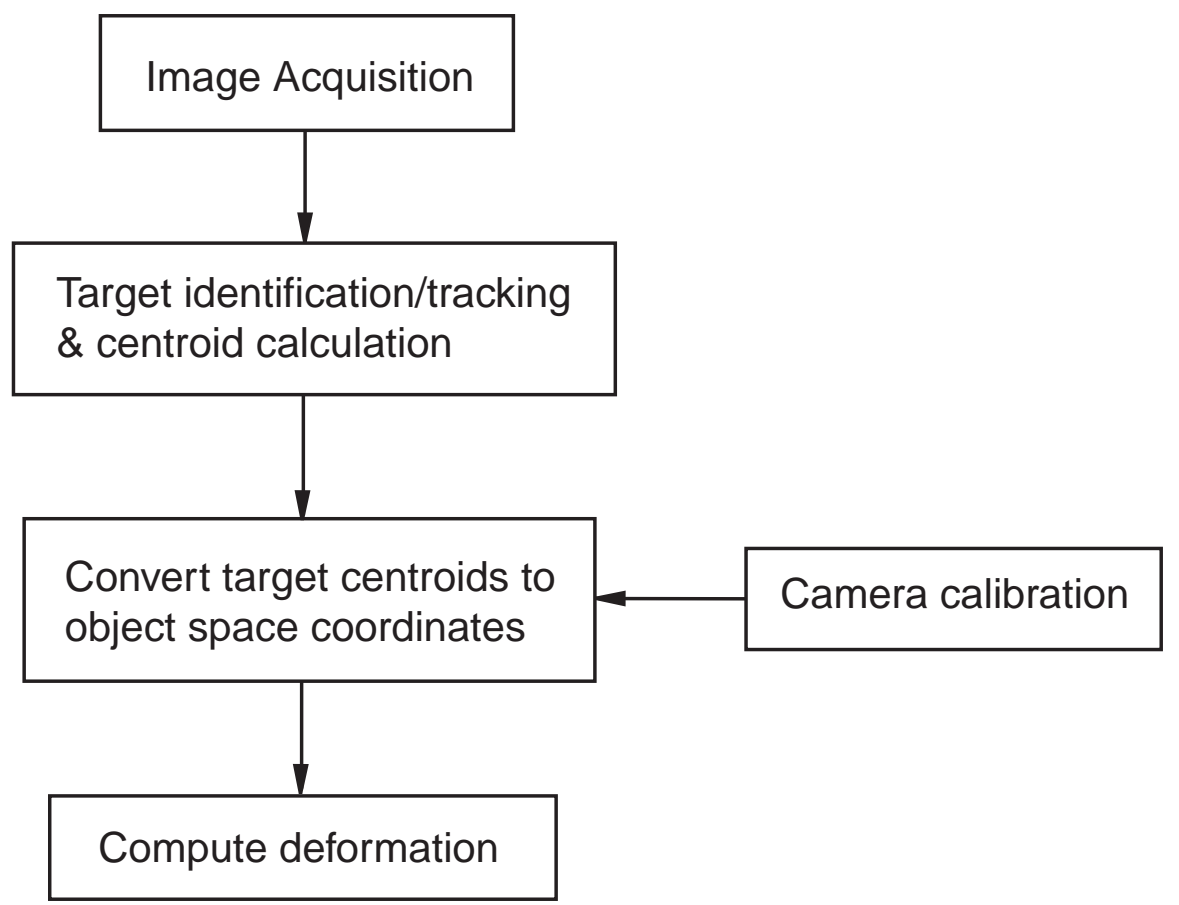

Figure 3. Flowchart of VMD data acquisition and processing 


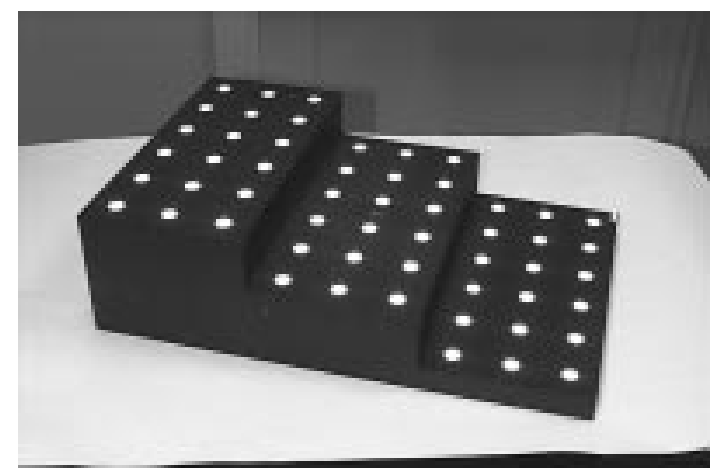

Figure 4. A step calibration target plate 


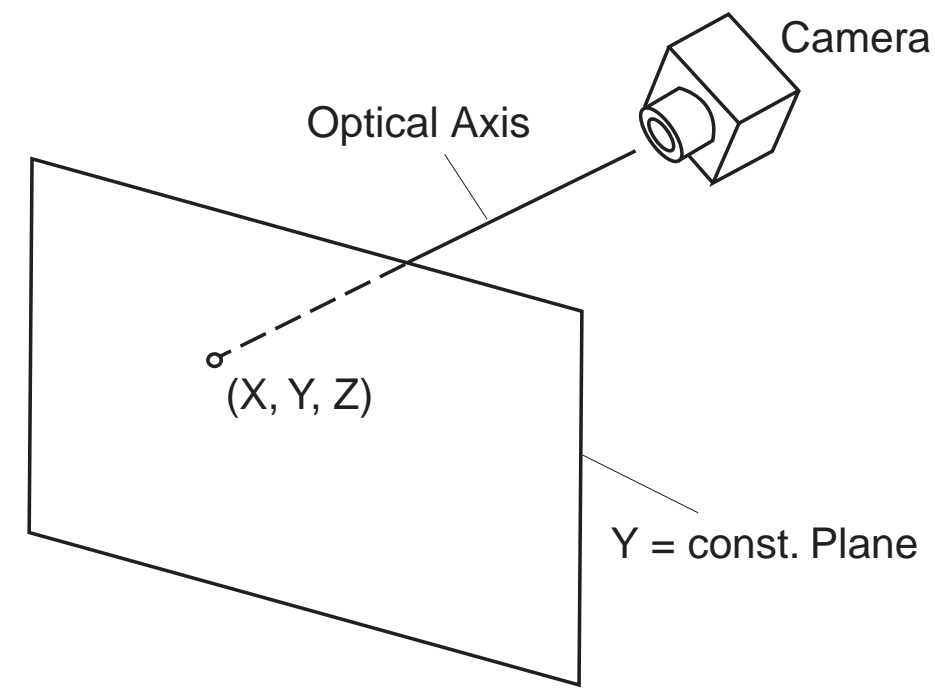

Figure 5. Geometric illustration of the single-camera solution. 


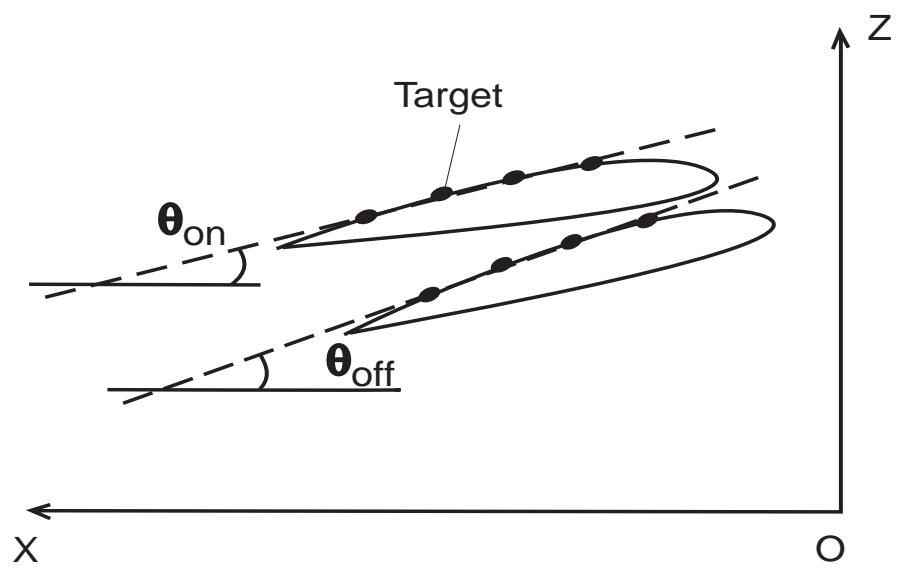

Figure 6. Wing deformation at a spanwise location. 


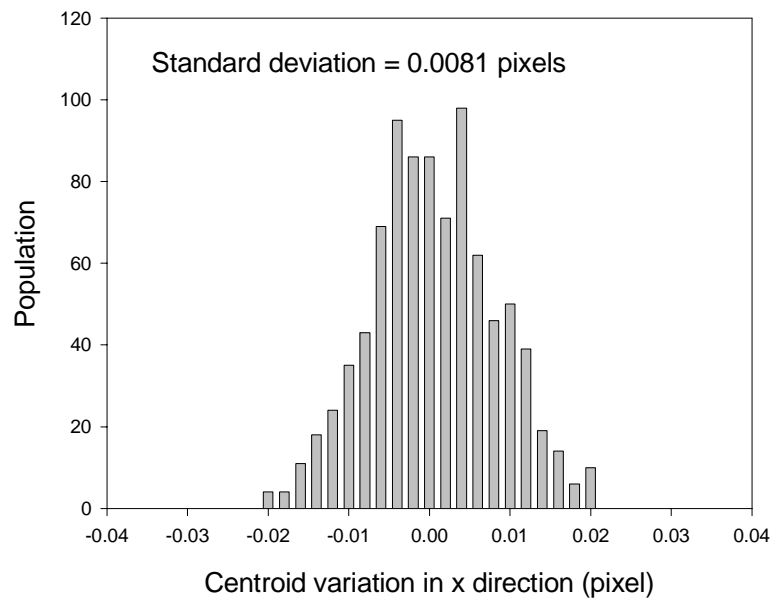

(a)

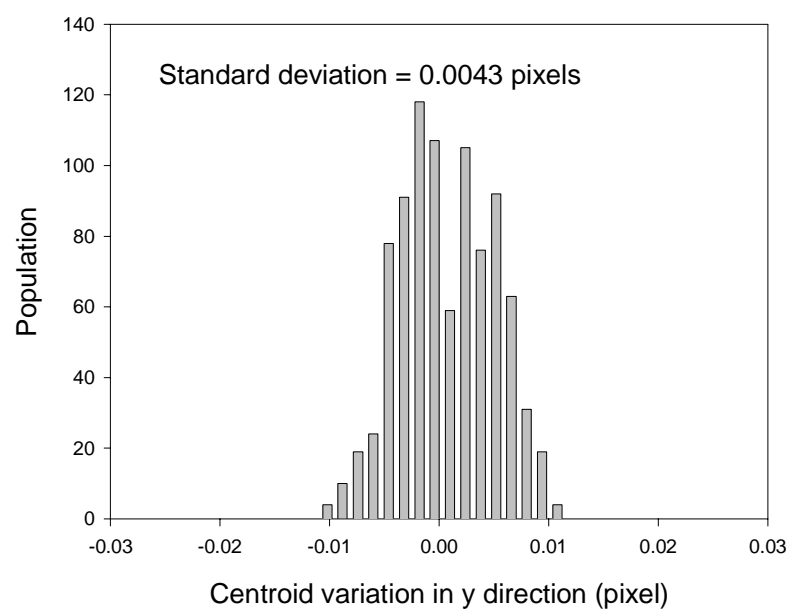

(b)

Figure 7. Histograms of centroid variation.

(a) Horizontal direction $\mathrm{x}$, (b) Vertical direction $\mathrm{y}$. 


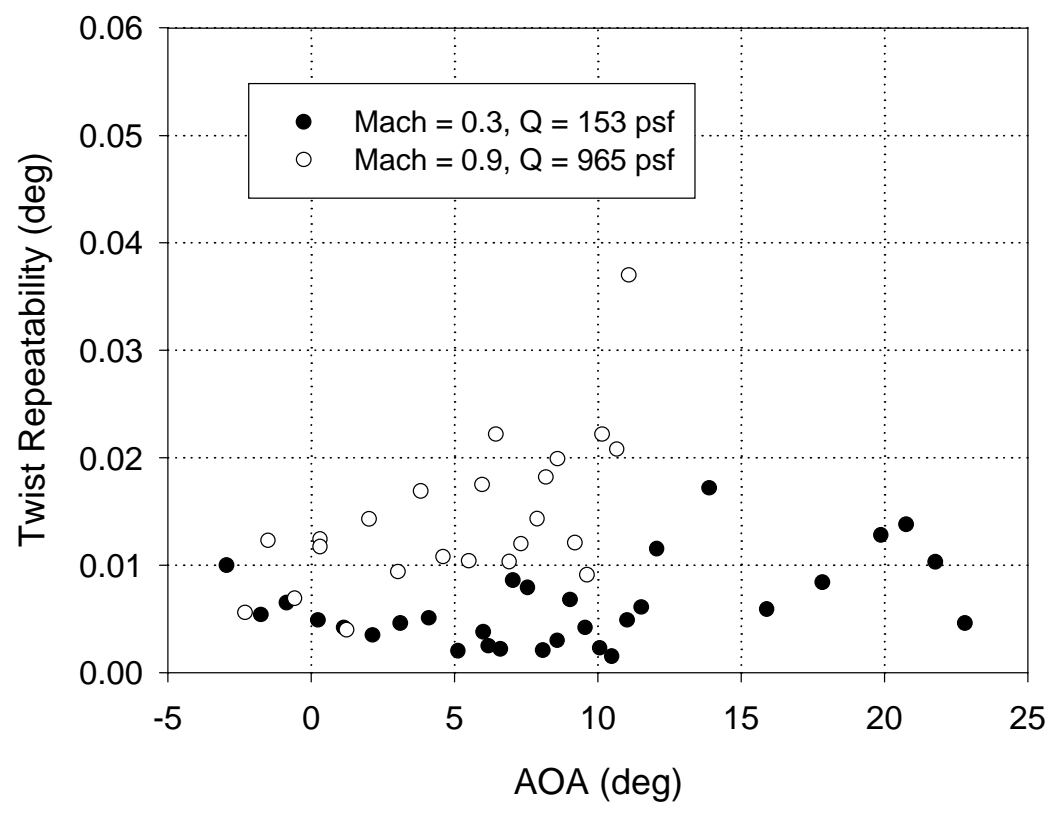

Figure 8. One sigma repeatability for air runs in NTF at two different Mach numbers and dynamic pressures at a wing spanwise location $\eta=0.922$. 


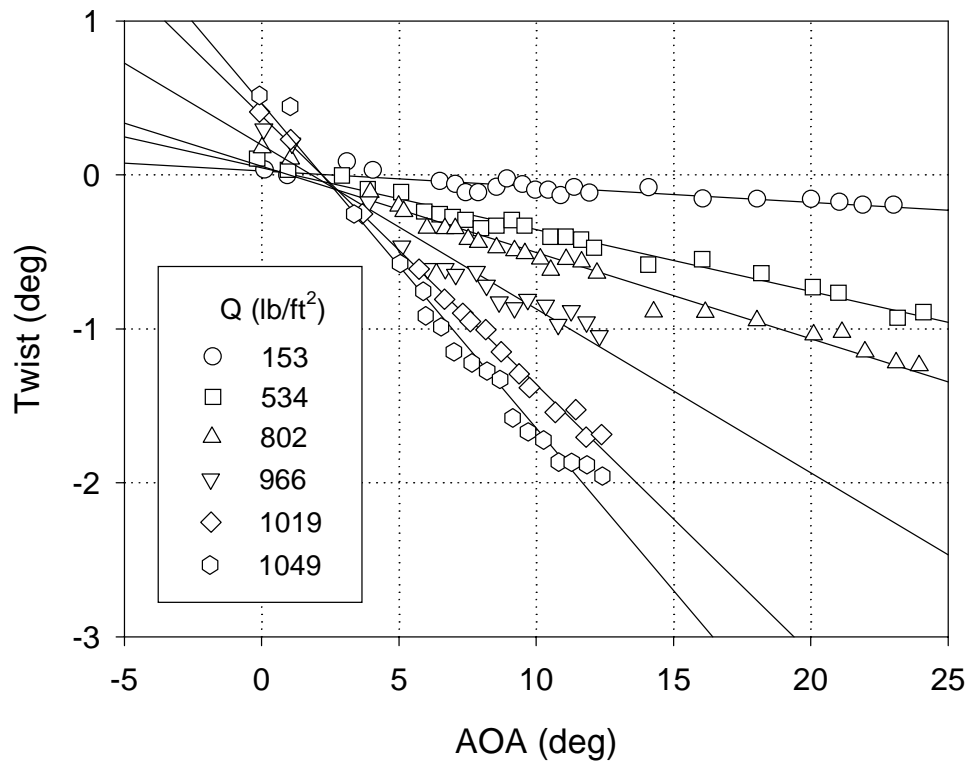

Figure 9. Typical wing twist of a HSR model at varying dynamic pressures for $\eta=0.922$. Measurements were carried out in NTF at NASA Langley. 


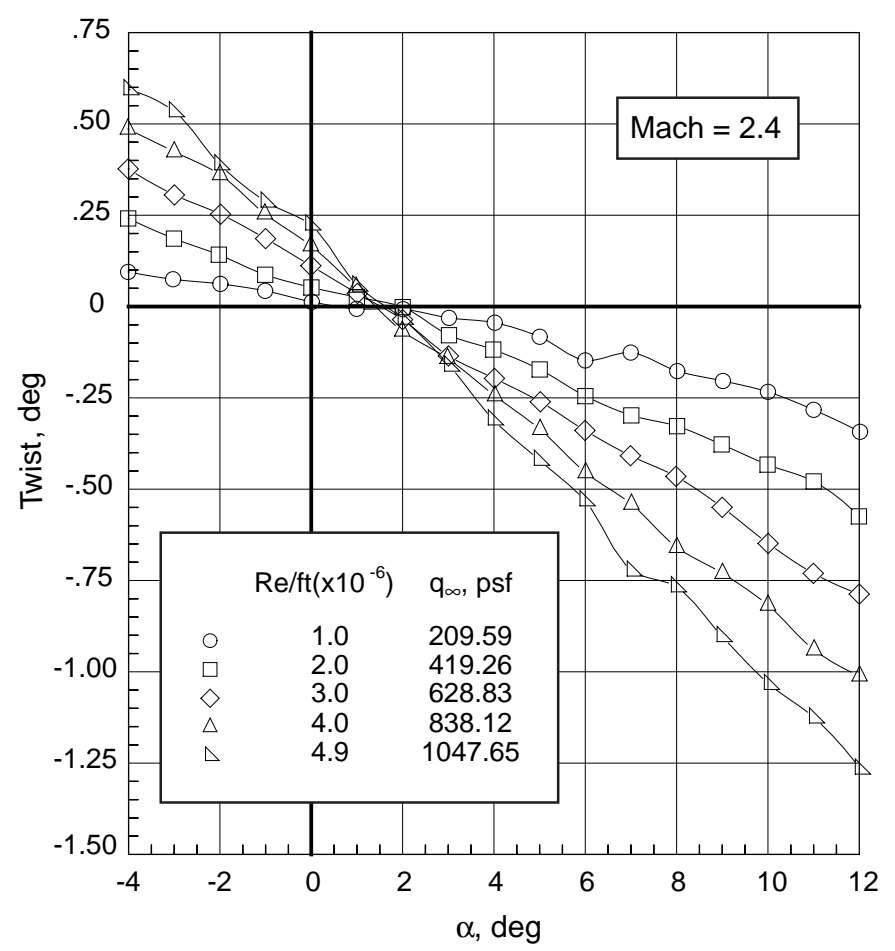

(a)

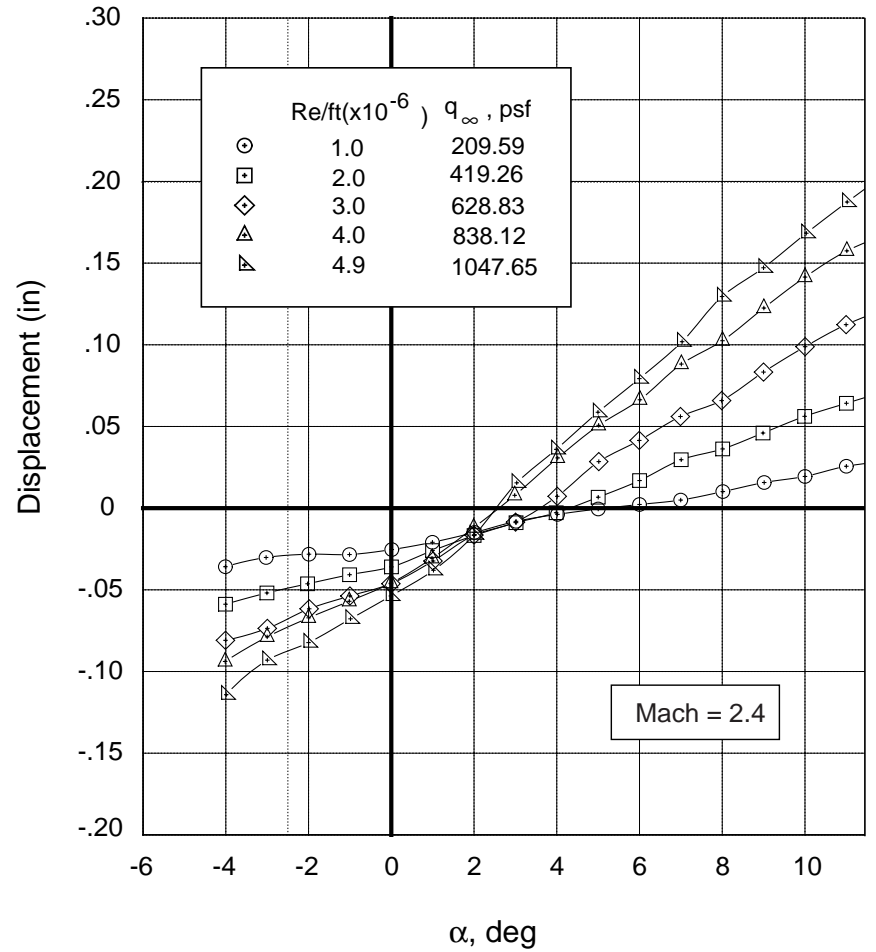

(b)

Figure 10. Typical wing twist of a HSR NCV model at different dynamic pressures for $\eta=0.922$. Measurements were carried out in UPWT at NASA Langley. 


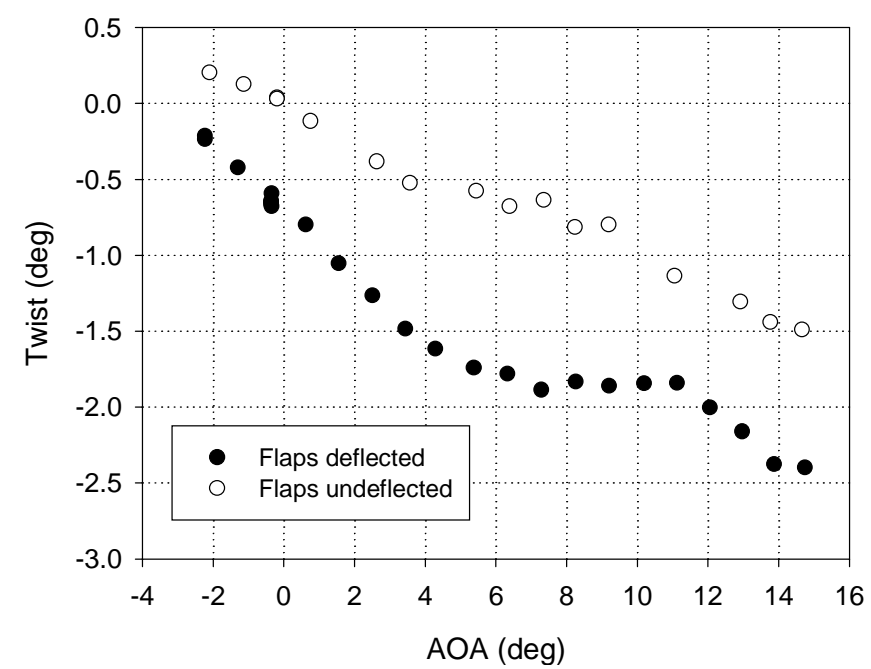

(a)

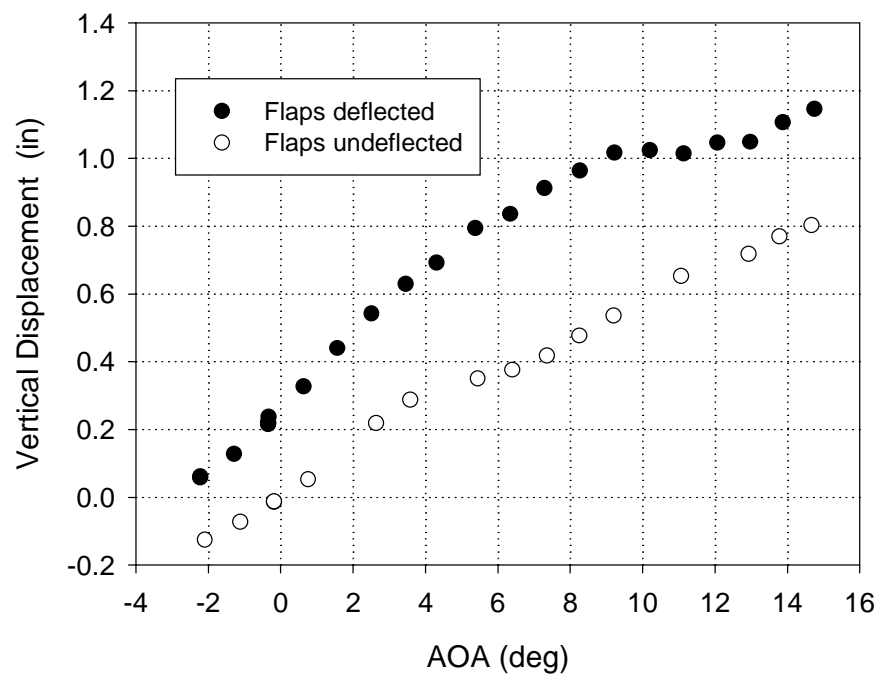

(b)

Figure 11. Twist and displacement of a $4 \%$ arrow wing of a low speed high lift model at Mach $=0.225$, $\operatorname{Re}=8.51 \times 10^{6}$, and $\mathrm{Q}=435 \mathrm{psf}$. Measurements were conducted at the Ames 12-Ft pressure tunnel. 


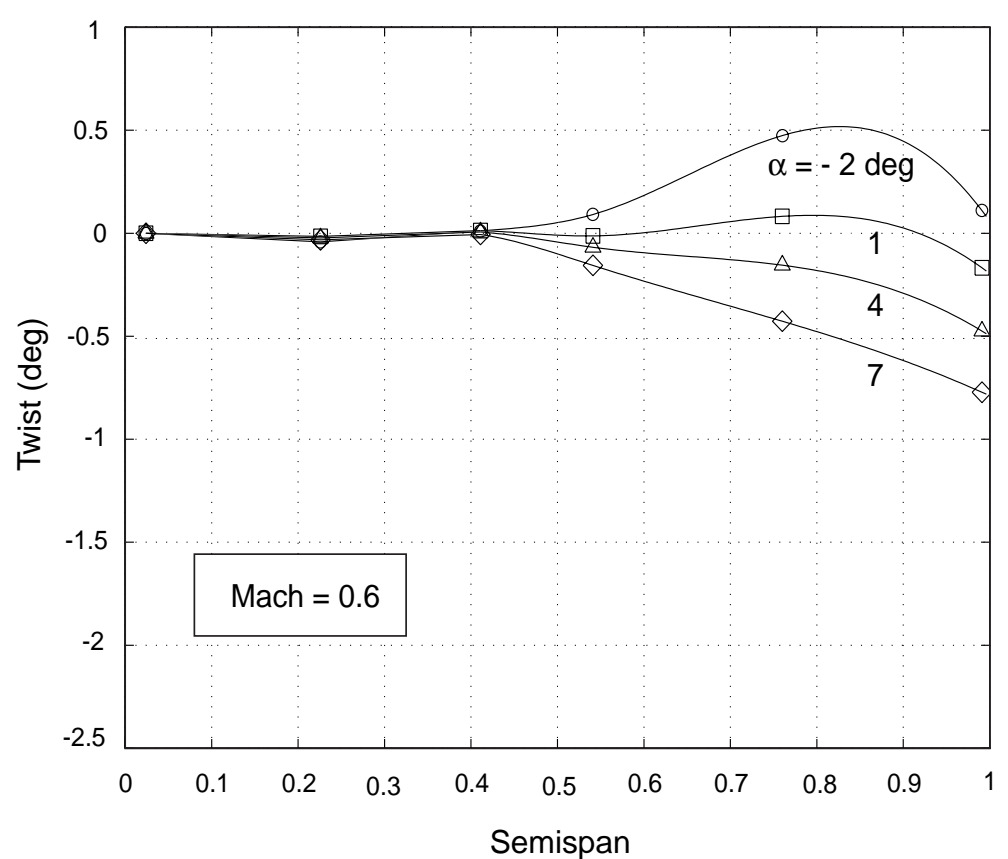

(a)

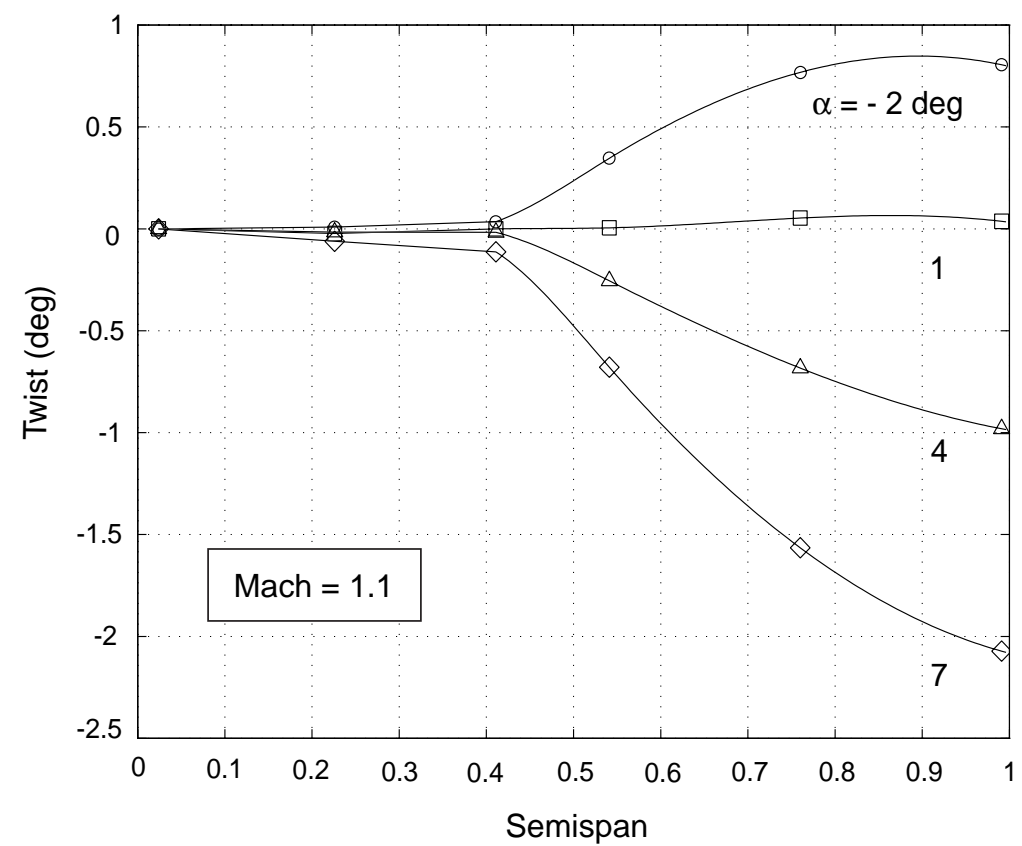

(b)

Figure 12. Spanwise twist distributions of a HSR TCA model at Mach $=0.6$ and $\mathrm{Mach}=1.1$. Measurements were made in the $16 \mathrm{Ft}$ Transonic Tunnel. 


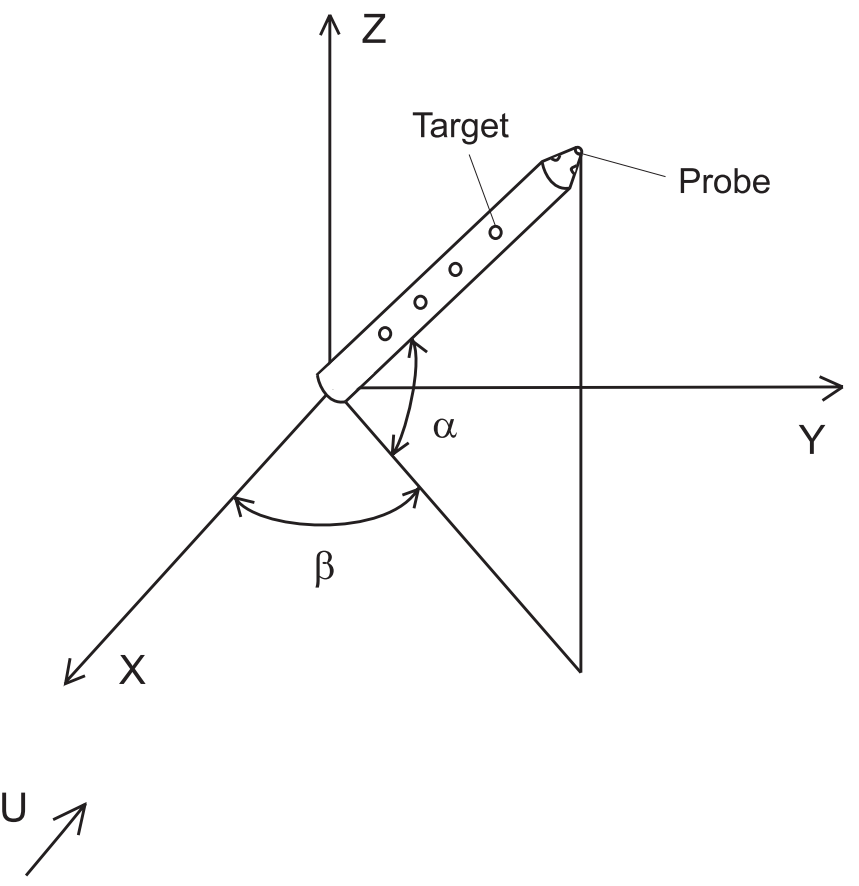

Figure 13. Probe orientation angles and coordinate system 

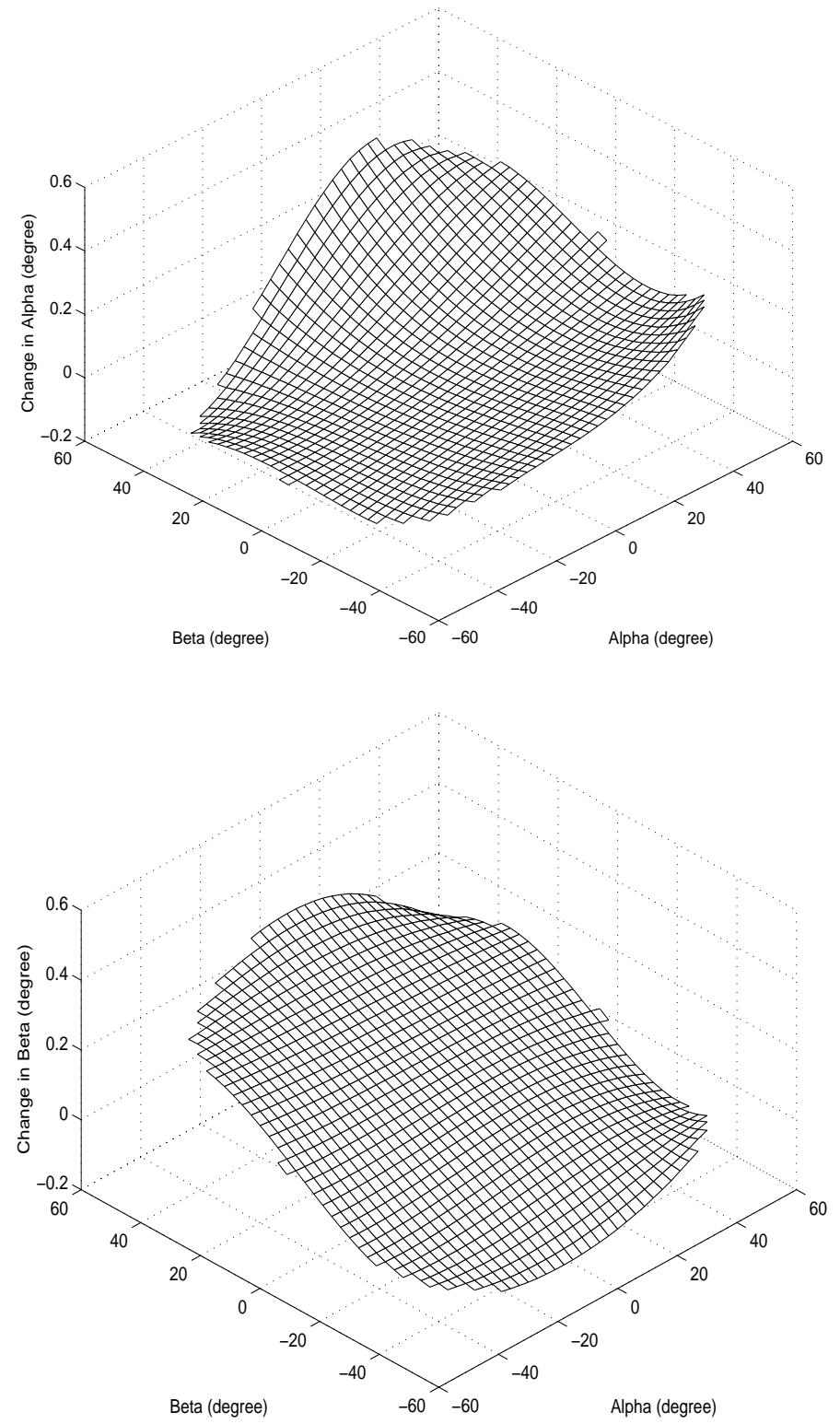

Figure 14. Distributions of probe orientation angle deviations from the rotational stage readings at Mach 0.5 and total pressure of 60 psi. 


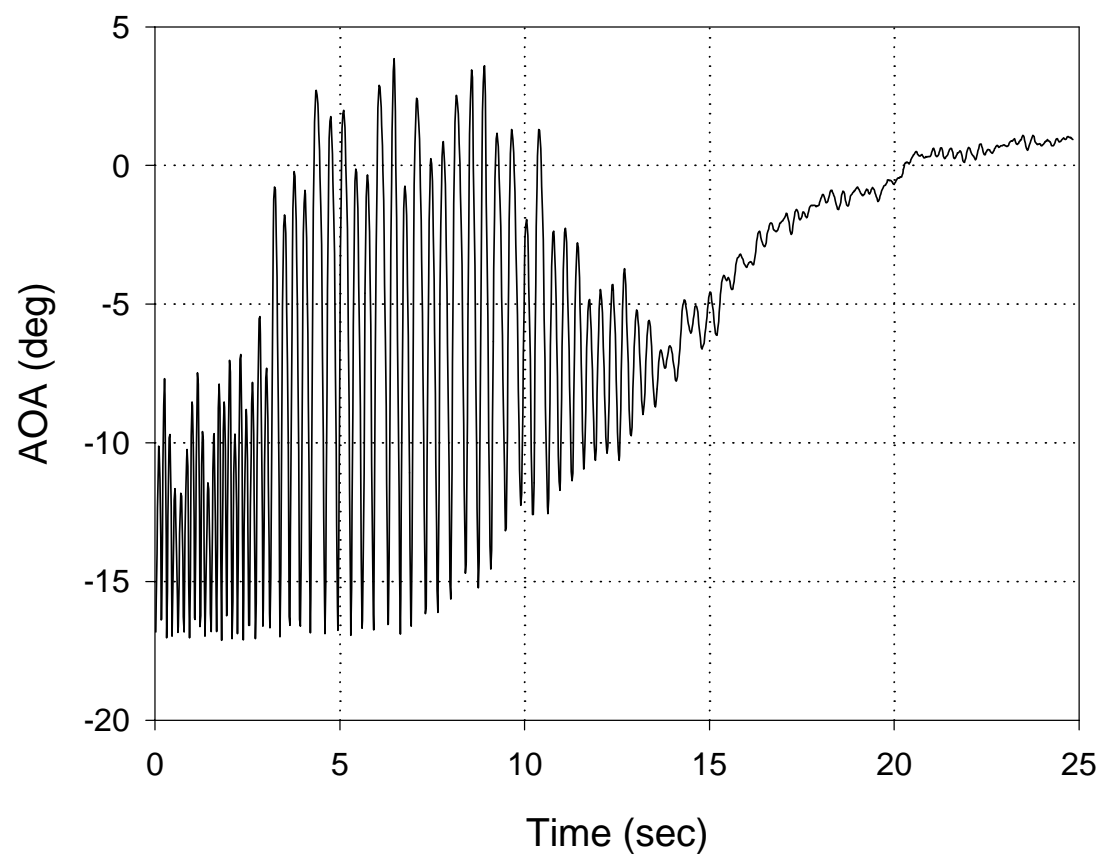

Figure 15. Time history of the angle-of-attack of an airfoil in an aeroelastic experiment. 